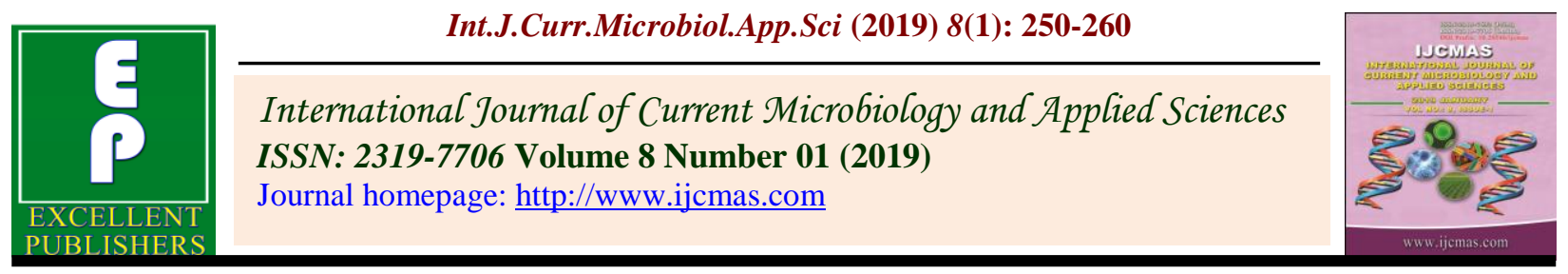

Original Research Article https://doi.org/10.20546/ijcmas.2019.801.028

\title{
Assessment of Genetic Diversity in Indian Common Bean Germplasm for Yield Traits
}

\author{
S. Sharma*, H.K. Chaudhary, A. Pathania and S. Thakur
}

Department of Crop Improvement, CSKHPKV, Palampur, Himachal Pradesh-176062, India

*Corresponding author:

\section{A B S T R A C T}

\begin{tabular}{|l|}
\hline Keyw or d s \\
Divergence, \\
Genetic variability, \\
Common bean \\
\hline Article Info \\
\hline Accepted: \\
04 December 2018 \\
Available Online: \\
10 January 2019 \\
\hline \hline
\end{tabular}

$\mathrm{D}^{2}$ statistics is a powerful tool for estimating genetic diversity among different genotypes for hybridization programme. On the basis of $\mathrm{D}^{2}$ values, the 169 genotypes were grouped into VIII clusters. Cluster II was the largest consisting of sixty two genotypes viz., KRC-2, K-326, HPK-322(2), HPR-396, VLF-106, K-255, KR-110, KR-249, K-249, VL-63, Palchan Local, Mani Rajma, Palchan kath, AK-40, HPR-80, HPR-24, HPR-38, AK-65, HPR-214, KR-296, HPR-8, KR-56-1, KR-118-1,KRC-16, KR-238, KR-155-3, KR-293, KR-52-2, KR-48-1, HUG-33, K-38, HPR-293, EC-84462, KR-256, AK-4, K-319, KRC12, KR-35, KRC-9, KR-175-1, KR-205, KR-96, KRC-22, Beeses 3 white, KR-171, K296, Premiere, KR-111, KR-53-2, KR-66-2, KR-24, KR-131, KR-240, KR-82, Ribba Local, R-10-457, KR-196, SR-1-6, SR-6-11, Jawala, Baspa. The next largest is clusters IV, followed cluster VII, V, VI, III, I each containing 42, 29, 16,12, 1 respectively. The assessment of genetic diversity helps in reducing the number of breeding lines from the large germplasm.

\section{Introduction}

Common bean (Phaseolus vulgaris L.; $2 n=2 x=22$ ) is a predominantly self-pollinated crop plant mainly originated in Latin America, probably Central Mexico and Guatemala. From Latin America, Spanish and Portuguese spreaded it into Europe, Africa and other parts of the World (Gepts and Bliss, 1988; Gepts et al., 1988; Zeven, 1997; Zeven et al., 1999). Nowadays, it is widely cultivated in the tropics, subtropics and temperate regions. Roughly $30 \%$ of common bean production in the world comes from Latin American countries. Due to its nutritive components, it is one of the 10 most important crops of the world. In India, common bean is known as 'Rajmash' and 'Frash bean' (green bean) and grows during summer and the winter in hilly areas of Himachal Pradesh, Jammu and Kashmir and North-Eastern states. In autumn, it is grown in parts of Uttar Pradesh, Maharashtra, Karnataka, and Andhra Pradesh. In Northern Indian plains, it is also cultivated on a limited scale as autumn or spring crop, because of its susceptibility to extreme temperatures. In India, the area under common bean cultivation is 9700 million ha as compared to 27,086 million ha all over the world, while its production is 4340 million 
tonnes as compared to 18,943 million tonnes in the world (FAO).

In India, common bean is known by the names of 'Rajmash' and 'Frash bean (green bean)' and grows during the summer and Genetic diversity plays an important role in plant breeding either to exploit heterosis or to generate productive recombinants. The choice of parents is of paramount importance in breeding programme. So, the knowledge of genetic diversity and relatedness in the germplasm is a prerequisite for crop improvement programmes. Reduction in the genetic variability makes the crops increasingly vulnerable to diseases and adverse climatic changes. So precise information on the nature and degree of genetic diversity present in collections from its principal areas of cultivation would help to select parents for evolving superior varieties. For the genetic amelioration of this crop, diverse genotypes from the existing germplasm should be selected and used in further breeding programme. $\mathrm{D}^{2}$ statistics is a powerful tool for estimating genetic diversity among different genotypes for hybridization programme. The assessment of genetic diversity helps in reducing the number of breeding lines from the large germplasm and the progenies derived from diverse parents are expected to show a broad spectrum of genetic variability and provide better scope to isolate superior recombinants.

\section{Materials and Methods}

The present investigation was carried out at the Experimental Farm CSK HPKV, Mountain Agricultural Research and Extension Centre (MAREC), Sangla, Distt. Kinnaur. The experimental material for the present study comprised of 165 local landraces of rajmash and 4 checks G19833 (A1), G4494 (A2) from Andean gene pool and DOR 364 (M1), ICAPIJAO (M2) from Mesoamerican gene pool of Rajmash ( Phaseolus vulgaris L.). These landraces along with checks were evaluated for different morphological and agronomic traits in Simple Lattice Design of $13 \times 13$ with two replications during kharif 2015. Two rows of each entry were grown in $1 \mathrm{~m}$ length with row-to-row and plant-to-plant distance of $50 \mathrm{~cm}$ and $5 \mathrm{~cm}$, respectively. Recommended package of practices were followed for raising the crop. Details of landraces used for the present study as given in table 1 .

\section{Observations recorded}

Observations were recorded for both qualitative traits as well as quantitative traits ( viz., Days to flowering, Days to maturity, Plant height $(\mathrm{cm})$, Branches per plant, Number of pods per plant, Pod length $(\mathrm{cm})$, Number of seeds per pod, Biological yield per plant $(\mathrm{g})$, Seed yield per plant (g), Harvest index (\%) and 100-seed weight (g) on five randomly selected plants per replication for all the genotypes except for days to flowering and days to maturity which was recorded on plot basis.

\section{Statistical methods}

Statistical analysis of the data was done as per Mahalanobis (1936) and using $\mathrm{D}^{2}$ values, different genotypes were grouped into various clusters following Tocher's method as suggested by Rao (1953). Cluster means of common bean genotypes falling under different clusters in individuals as well as combined over environments were also calculated.

On the basis of D2 values, the 169 genotypes were grouped into VIII clusters (Table 2). Cluster II was the largest consisting of sixty two genotypes viz., KRC-2, K-326, HPK322(2), HPR-396, VLF-106, K-255, KR-110, KR-249, K-249, VL-63, Palchan Local,, Mani 
Rajma, Palchan kath, AK-40, HPR-80, HPR24, HPR-38, AK-65, HPR-214,KR-296, HPR8, KR-56-1, KR-118-1,KRC-16, KR-238, KR155-3, KR-293, KR-52-2, KR-48-1, HUG-33, K-38, HPR-293, EC-84462, KR-256, AK-4, K-319, KRC-12, KR-35, KRC-9, KR-175-1, KR-205, KR-96, KRC-22, Beeses 3 white, KR-171, K-296, Premiere, KR-111, KR-53-2,
KR-66-2，KR-24，KR-131，KR-240，KR-82, Ribba Local, R-10-457, KR-196, SR-1-6, SR6-11, Jawala, Baspa. The next largest is clusters IV, followed cluster VII, V, VI, III, I each containing 42, 29, 16,12, 1 respectively. Sharma et al. (2009) also used D2 statistics to study genetic diversity and grouped common bean germplasm into six clusters.

Table.1 Details of material used in the present study

\begin{tabular}{|c|c|c|}
\hline S.No. & Local Landraces & Accession No. \\
\hline 1 & KR-202-I & AC-1 \\
\hline 2 & KR-77 & $\mathrm{AC}-2$ \\
\hline 3 & KR-93 & $\mathrm{AC}-3$ \\
\hline 4 & KRC-21 & AC-4 \\
\hline 5 & IC 313623 & AC-5 \\
\hline 6 & AK 61 & AC-6 \\
\hline 7 & $\mathrm{~K}-326$ & $\mathrm{AC}-7$ \\
\hline 8 & HPK-322(2) & AC- 8 \\
\hline 9 & $\mathrm{~K}-258$ & AC-9 \\
\hline 10 & K-243 & AC-10 \\
\hline 11 & HPR-396 & $\mathrm{AC}-11$ \\
\hline 12 & HPR-415 & AC-12 \\
\hline 13 & VLF-106 & $\mathrm{AC}-13$ \\
\hline 14 & $\mathrm{~K}-255$ & AC-14 \\
\hline 15 & HPR-432 & $\mathrm{AC}-15$ \\
\hline 16 & KR-110 & AC-16 \\
\hline 17 & KR-249 & $\mathrm{AC}-17$ \\
\hline 18 & KR-94 & AC-18 \\
\hline 19 & Sarahan Local & AC-19 \\
\hline 20 & KR-126 & AC-20 \\
\hline 21 & AK-48 & $\mathrm{AC}-21$ \\
\hline 22 & EC-316088 & $\mathrm{AC}-22$ \\
\hline 23 & $\mathrm{~K}-249$ & $\mathrm{AC}-23$ \\
\hline 24 & VL-63 & AC-24 \\
\hline 25 & Palchan Local & $\mathrm{AC}-25$ \\
\hline 26 & Palchan Kath & AC-26 \\
\hline 27 & Mani Rajma & $\mathrm{AC}-27$ \\
\hline 28 & AK-23 & AC-28 \\
\hline 29 & Palchan Kath & AC-29 \\
\hline 30 & $\mathrm{AK}-40$ & AC-30 \\
\hline 31 & AK-64 & AC-31 \\
\hline 32 & HPR-80 & AC-32 \\
\hline 33 & HPR-24 & AC-33 \\
\hline 34 & HPR-38 & AC-34 \\
\hline 35 & AK-65 & AC-35 \\
\hline 36 & AK-73 & AC-36 \\
\hline 37 & HPR-214 & AC-37 \\
\hline 38 & Rakhcham Local & AC-38 \\
\hline 39 & $\mathrm{~K}-163$ & AC-39 \\
\hline 40 & HPR-16 & AC- 40 \\
\hline 41 & KRC-11 & AC-41 \\
\hline 42 & KR-253-A & AC-42 \\
\hline 43 & KR-273 & AC-43 \\
\hline 44 & KR-175 & AC-44 \\
\hline 45 & KR-296 & $\mathrm{AC}-45$ \\
\hline 46 & R-10-453 & AC-46 \\
\hline 47 & KR-176 & AC-47 \\
\hline
\end{tabular}


Int.J.Curr.Microbiol.App.Sci (2019) 8(1): 250-260

\begin{tabular}{|c|c|c|}
\hline 48 & AK-6 & AC- -48 \\
\hline 49 & Dalhera Local & AC-49 \\
\hline 50 & AK-37 & AC-50 \\
\hline 51 & AK-16 & AC-51 \\
\hline 52 & HPR-8 & AC-52 \\
\hline 53 & KR-40 & AC-53 \\
\hline 54 & KR-56-1 & AC-54 \\
\hline 55 & KR-280 & $\mathrm{AC}-55$ \\
\hline 56 & KR-118-1 & AC-56 \\
\hline 57 & KRC-16 & AC-57 \\
\hline 58 & KR-51 & AC-58 \\
\hline 59 & KR-238 & AC-59 \\
\hline 60 & KR-155-3 & AC-60 \\
\hline 61 & KR-293 & AC-61 \\
\hline 62 & KR-52-2 & AC-62 \\
\hline 63 & KR-48-1 & AC-63 \\
\hline 64 & HUG-33 & AC-64 \\
\hline 65 & $\mathrm{~K}-38$ & AC-65 \\
\hline 66 & KRC-18 & AC-66 \\
\hline 67 & HPR-293 & AC-67 \\
\hline 68 & HPR-44 & AC-68 \\
\hline 69 & EC-84462 & AC-69 \\
\hline 70 & K-158 & AC-70 \\
\hline 71 & KR-142 & AC-71 \\
\hline 72 & AK-77 & AC-72 \\
\hline 73 & K-284 & $\mathrm{AC}-73$ \\
\hline 74 & KR-256 & AC-74 \\
\hline 75 & AK-4 & $\mathrm{AC}-75$ \\
\hline 76 & AK-82 & AC-76 \\
\hline 77 & AK-57 & AC-77 \\
\hline 78 & Saimulchan Local & AC-78 \\
\hline 79 & KR-32 & AC-79 \\
\hline 80 & KR-9 & AC-80 \\
\hline 81 & KR-142-1 & AC-81 \\
\hline 82 & KR-227 & AC- 82 \\
\hline 83 & KR-169 & $\mathrm{AC}-83$ \\
\hline 84 & KR-133 & AC-84 \\
\hline 85 & KRC-4 & AC- 85 \\
\hline 86 & AK-68-A & AC-86 \\
\hline 87 & $\mathrm{~K}-29$ & AC-87 \\
\hline 88 & K-319 & AC- 88 \\
\hline 89 & $\mathrm{~K}-85$ & AC-89 \\
\hline 90 & AK-36 & AC-90 \\
\hline 91 & KR-6 & AC-91 \\
\hline 92 & AK-3 & AC-92 \\
\hline 93 & HPR-159 & AC-93 \\
\hline 94 & $\mathrm{~K}-214$ & AC-94 \\
\hline 95 & K-289 & AC-95 \\
\hline 96 & AK-53 & AC-96 \\
\hline 97 & AK-50 & AC-97 \\
\hline 98 & KR-70-3 & AC-98 \\
\hline 99 & $\mathrm{~K}-16$ & AC-99 \\
\hline 100 & K-264 & AC-100 \\
\hline 101 & K-191 & AC-101 \\
\hline 102 & KRC-12 & AC-102 \\
\hline 103 & KRC-241 & AC-103 \\
\hline 104 & KR-242-1 & AC-104 \\
\hline 105 & KR-243 & AC-105 \\
\hline 106 & KR-307 & AC-106 \\
\hline 107 & KR-134 & AC-107 \\
\hline 108 & KR-35 & AC-108 \\
\hline 109 & KR-216-I & AC-109 \\
\hline 110 & KRC-9 & $\mathrm{AC}-110$ \\
\hline
\end{tabular}


Int.J.Curr.Microbiol.App.Sci (2019) 8(1): 250-260

\begin{tabular}{|c|c|c|}
\hline 111 & AK-66 & AC-111 \\
\hline 112 & AK-44 & AC-112 \\
\hline 113 & AK-39 & $\mathrm{AC}-113$ \\
\hline 114 & HPR-139 & AC-114 \\
\hline 115 & KR-175-1 & AC- -115 \\
\hline 116 & KR-205 & $\mathrm{AC}-116$ \\
\hline 117 & KR-96 & AC- -117 \\
\hline 118 & HPR-21 & AC- -118 \\
\hline 119 & K-254 & AC-119 \\
\hline 120 & K-168 & AC- -120 \\
\hline 121 & HPR-360 & $\mathrm{AC}-121$ \\
\hline 122 & Kalera Local & AC-122 \\
\hline 123 & AK-1 & AC-123 \\
\hline 124 & HPR-84 & AC-124 \\
\hline 125 & HPR-300 & AC- -125 \\
\hline 126 & KRC-22 & AC-126 \\
\hline 127 & KR-70-3 & AC-127 \\
\hline 128 & KR-72 & AC- -128 \\
\hline 129 & KR-117 & AC-129 \\
\hline 130 & KR-192 & AC- -130 \\
\hline 131 & KR-276 & AC-131 \\
\hline 132 & Beeses 3 White & AC-132 \\
\hline 133 & HPR-339 & AC-133 \\
\hline 134 & HPR-224 & AC-134 \\
\hline 135 & KR-88 & $\mathrm{AC}-135$ \\
\hline 136 & KR-247 & AC-136 \\
\hline 137 & KR-135 & AC-137 \\
\hline 138 & KR-89 & AC-138 \\
\hline 139 & KR-161 & AC-139 \\
\hline 140 & KR-171 & AC- 140 \\
\hline 141 & K-296 & AC-141 \\
\hline 142 & Premiere & $\mathrm{AC}-142$ \\
\hline 143 & HPR-54 & AC-143 \\
\hline 144 & AK-89 & AC-144 \\
\hline 145 & AK-87 & AC- -145 \\
\hline 146 & KR-111 & AC-146 \\
\hline 147 & KR-53-2 & AC- -147 \\
\hline 148 & KR-66-2 & AC- -148 \\
\hline 149 & KR-29-2 & AC-149 \\
\hline 150 & KR-292 & $\mathrm{AC}-150$ \\
\hline 151 & KR-24 & AC-151 \\
\hline 152 & KR-62-2 & AC-152 \\
\hline 153 & AK-62 & AC- -153 \\
\hline 154 & AK-42 & AC-154 \\
\hline 155 & KR-131 & AC -155 \\
\hline 156 & KR-240 & AC-156 \\
\hline 157 & KR-82 & AC-157 \\
\hline 158 & Ribba Local & AC- -158 \\
\hline 159 & R-10-57 & AC-159 \\
\hline 160 & KR-196 & AC-160 \\
\hline 161 & SR-1-6 & AC-161 \\
\hline 162 & SR-6-11 & AC-162 \\
\hline 163 & Kailash & AC-163 \\
\hline 164 & Jawala & AC-164 \\
\hline 165 & Baspa & $\mathrm{AC}-165$ \\
\hline 166 & G19833 & A1 \\
\hline 167 & G4494 & $\mathrm{A} 2$ \\
\hline 168 & DOR364 & M1 \\
\hline 169 & ICA PIJAO & M2 \\
\hline
\end{tabular}


Table.2 Distribution of rajmash genotypes into different clusters

\begin{tabular}{|c|c|c|}
\hline & Number of genotypes & Genotypes \\
\hline I & 1 & KR-202-1 \\
\hline II & 62 & $\begin{array}{l}\text { KRC-21, K-326, HPK-322(2), HPR-396, VLF-106, K-255, } \\
\text { KR-110, KR-249, K-249, VL-63, Palchan Local, Palchan } \\
\text { Kath, Mani Rajma, AK-40, HPR-80, HPR-24, HPR-38, } \\
\text { AK-65, HPR-214, KR-296, HPR-8, KR-56-1, KR-118-1, } \\
\text { KRC-16, KR-238, KR-155-3, KR-293, KR-52-2, KR-48-1, } \\
\text { HUG-33, K-38, HPR-293, EC-84462, KR-256, AK-4, K- } \\
\text { 319, KRC-12, KR-35, KRC-9, KR-175-1, KR-205, KR-96, } \\
\text { KRC-22, Beese 3 white, KR-171, K-296, Premiere, KR- } \\
\text { 111, KR-53-2, KR-66-2, KR-24, KR-131, KR-240, KR-82, } \\
\text { Ribba Local, R-10-457, KR-196, SR-1-6, SR-6-11, Jawala, } \\
\text { Baspa }\end{array}$ \\
\hline III & 6 & $\begin{array}{l}\text { KR-253-A, KR-273, KR-176, AK-6, Dalhera, Local, AK- } \\
37\end{array}$ \\
\hline IV & 42 & $\begin{array}{l}\text { KR-93, AK-61, K-258, K-243, KR-94, Sarahan Local, } \\
\text { KR-126, AK-23, KR-175, R-10-453, KR-40, KR-51, K- } \\
\text { 158, AK-77, KR-227, KR-133, AK-53, AK-50, K-16, K- } \\
\text { 264, KRC-242-1, KR-134, KR-216-I, K-254, Kalera } \\
\text { Local, HPR-84, HPR-300, KR-70-3, KR-72, KR-117, KR- } \\
\text { 192, KR-276, HPR-339, KR-247, KR-135, KR-161, KR- } \\
\text { 29-2, KR-292, AK-62, AK-42, DOR 364, ICAPIJAO }\end{array}$ \\
\hline $\mathrm{V}$ & 16 & $\begin{array}{l}\text { IC-313623, HPR-415, AK-64, AK-73, K-163, HPR-16, } \\
\text { AK-16, KR-280, K-284, HPR-159, K-214, K-191, K-168, } \\
\text { HPR-224, KR-88, Kailash }\end{array}$ \\
\hline VI & 12 & $\begin{array}{l}\text { AK-48, EC-316088, KR-142, AK-82, AK-57, KR-32, } \\
\text { KRC-241, HPR-360, AK-1, HPR-54, AK-89, AK-87 }\end{array}$ \\
\hline VII & 29 & $\begin{array}{l}\text { KR-77, HPR-432, Rakcham Local, KRC-11, KRC-18, } \\
\text { HPR-44, Saimulchan Local, KR-9, KR-142-1, KR-169, } \\
\text { KRC-4, AK-68-A, K-29, K-85, AK-36, KR-6, AK-3, K- } \\
\text { 289, KR-70-3, KR-243, AK-66, AK-44, AK-39, HPR-139, } \\
\text { HPR-21, KR-89, KR-62-2, G19833, G4494. }\end{array}$ \\
\hline VIII & 1 & KR-307 \\
\hline
\end{tabular}

Table.3 Average intra and inter-cluster distances among eight clusters

\begin{tabular}{|c|c|c|c|c|c|c|c|c|}
\hline Clusters & I & II & III & IV & V & VI & VII & VIII \\
\hline I & $\mathbf{0 . 0 0}$ & 161.36 & 92.31 & 149.99 & 135.53 & 134.63 & 149.52 & 153.89 \\
\hline II & & $\mathbf{1 6 . 6 6}$ & 82.14 & 30.90 & 37.45 & 58.36 & 28.96 & 69.04 \\
\hline III & & & $\mathbf{1 9 . 3 4}$ & 64.59 & 49.16 & 47.58 & 67.86 & 85.88 \\
\hline IV & & & & $\mathbf{1 5 . 1 9}$ & 21.43 & 29.90 & 25.45 & 71.60 \\
\hline V & & & & & $\mathbf{1 7 . 5 7}$ & 27.11 & 21.52 & 58.50 \\
\hline VI & & & & & & $\mathbf{1 9 . 6 5}$ & 41.02 & 72.99 \\
\hline VII & & & & & & & $\mathbf{1 5 . 6 8}$ & 48.58 \\
\hline VIII & & & & & & & & $\mathbf{0 . 0 0}$ \\
\hline
\end{tabular}

*Diagonal values are intra cluster distances 
Table.4 Cluster means of eight clusters for different traits of rajmash genotypes

\begin{tabular}{|l|l|l|l|l|l|l|l|l|l|l|l|}
\hline Traits & I & II & III & IV & V & VI & VII & VIII & Mean & $\begin{array}{l}\text { Maximu } \\
\text { m }\end{array}$ & $\begin{array}{l}\text { Minimu } \\
\text { m }\end{array}$ \\
\hline Plant height & 85.0 & 45.96 & 90.42 & 73.31 & 74.44 & 98.58 & 66.57 & 72.50 & 75.85 & 98.58 \\
\hline Branches/plant & 3.00 & 3.39 & 3.92 & 3.38 & 3.50 & 3.96 & 3.41 & 3.50 & 3.51 & 3.92 & 3.00 \\
\hline $\begin{array}{l}\text { No. of pods/ } \\
\text { plant }\end{array}$ & 15.00 & 10.97 & 24.04 & 11.62 & 15.22 & 15.41 & 11.40 & 10.00 & 14.21 & 24.04 & 10.00 \\
\hline Pod length & 9.40 & 10.18 & 9.53 & 9.68 & 10.41 & 10.55 & 10.90 & 13.80 & 10.56 & 13.80 & 9.40 \\
\hline No. of seed/pod & 6.50 & 4.79 & 5.75 & 4.92 & 5.22 & 4.63 & 4.36 & 4.00 & 5.02 & 6.50 & 4.00 \\
\hline Biological yield & 35.10 & 20.52 & 16.46 & 26.55 & 39.79 & 36.83 & 29.66 & 39.00 & 30.49 & 39.79 & 16.46 \\
\hline Harvest index & 11.80 & 41.78 & 45.71 & 38.90 & 44.57 & 40.46 & 40.31 & 52.58 & 39.51 & 52.58 & 11.80 \\
\hline 100 seed wt & 33.70 & 33.32 & 24.56 & 24.87 & 35.67 & 27.03 & 48.68 & 90.54 & 39.80 & 90.55 & 24.56 \\
\hline $\begin{array}{l}\text { Days to } \\
\text { flowering }\end{array}$ & 21.40 & 73.89 & 81.58 & 77.63 & 78.34 & 76.25 & 75.40 & 61.50 & 68.25 & 81.58 & 21.40 \\
\hline $\begin{array}{l}\text { Days to } \\
\text { maturity }\end{array}$ & 74.00 & 124.68 & 131.00 & 133.44 & 129.53 & 135.63 & 133.98 & 124.00 & 123.28 & 135.63 & 74.00 \\
\hline Seed yield & 140.00 & 8.28 & 7.37 & 10.27 & 17.67 & 14.96 & 11.92 & 20.50 & 28.87 & 140.00 & 7.37 \\
\hline
\end{tabular}

Table.5 Relative contribution (\%) of individual trait to the genetic divergence among rajmash genotypes

\begin{tabular}{|c|c|c|c|}
\hline S. No & Traits & No. of times ranked first & Contribution (\%) \\
\hline 1 & Plant height $(\mathrm{cm})$ & 684 & 38.14 \\
\hline 2 & Branches per plant & 0 & $0 *$ \\
\hline 3 & No. of pods per plant & 19 & 1.05 \\
\hline 4 & Pod Length (cm) & 1 & 0.05 \\
\hline 5 & No. of seeds per pod & 0 & $0 *$ \\
\hline 6 & Biological yield per plant (g) & 687 & $38.31 * *$ \\
\hline 7 & Seed yield per plant (g) & 104 & 5.80 \\
\hline 8 & Harvest Index (\%) & 12 & 0.66 \\
\hline 9 & 100 seed weight & 221 & 12.32 \\
\hline 10 & Days to flowering & 14 & 0.78 \\
\hline 11 & Days to maturity & 51 & 2.84 \\
\hline
\end{tabular}

\footnotetext{
*Minimum; **Maximum
} 
Fig.1 Dendrogram of rajmash genotypes generated using Mahalanobis D²-cluster analysis

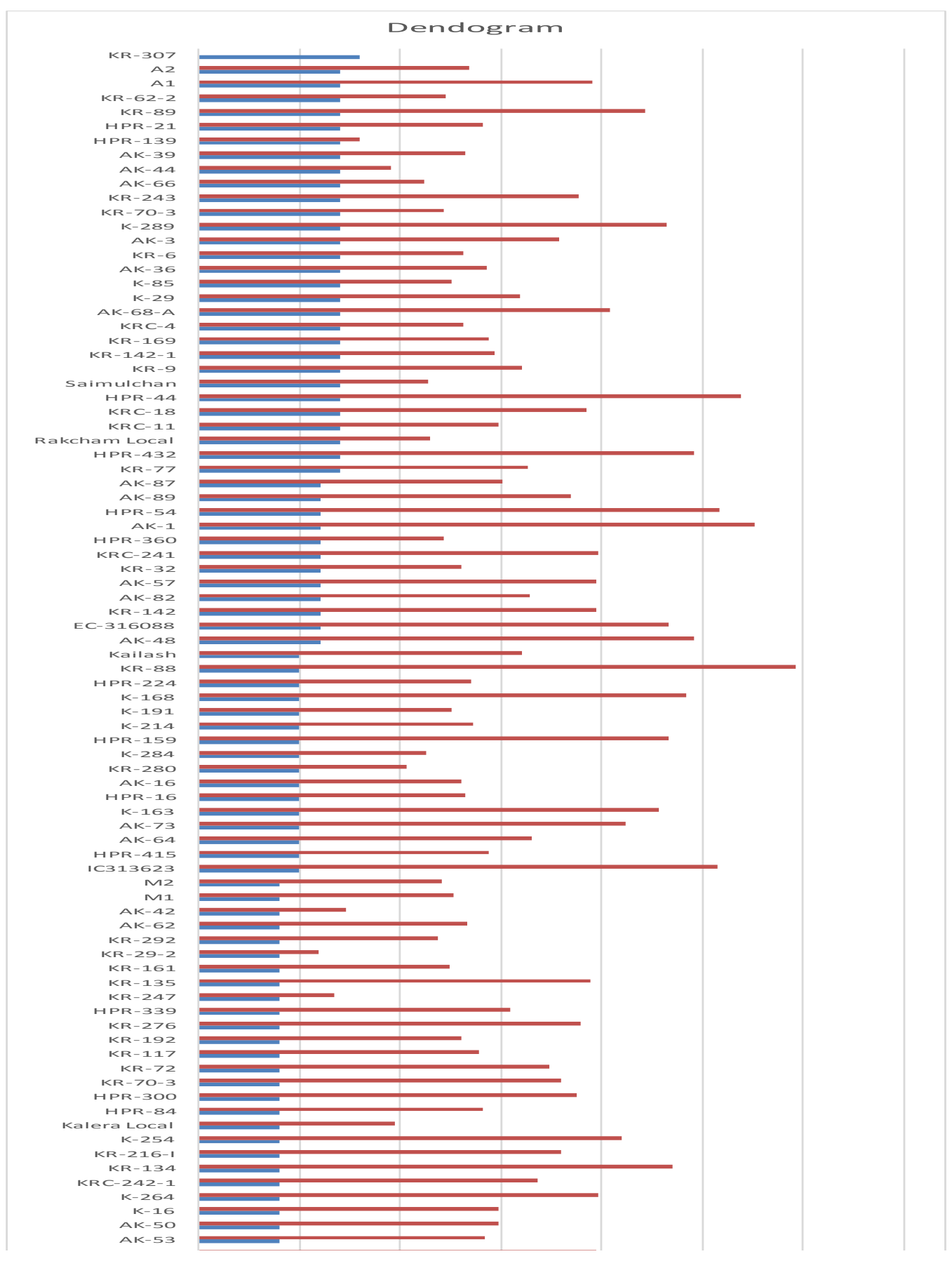




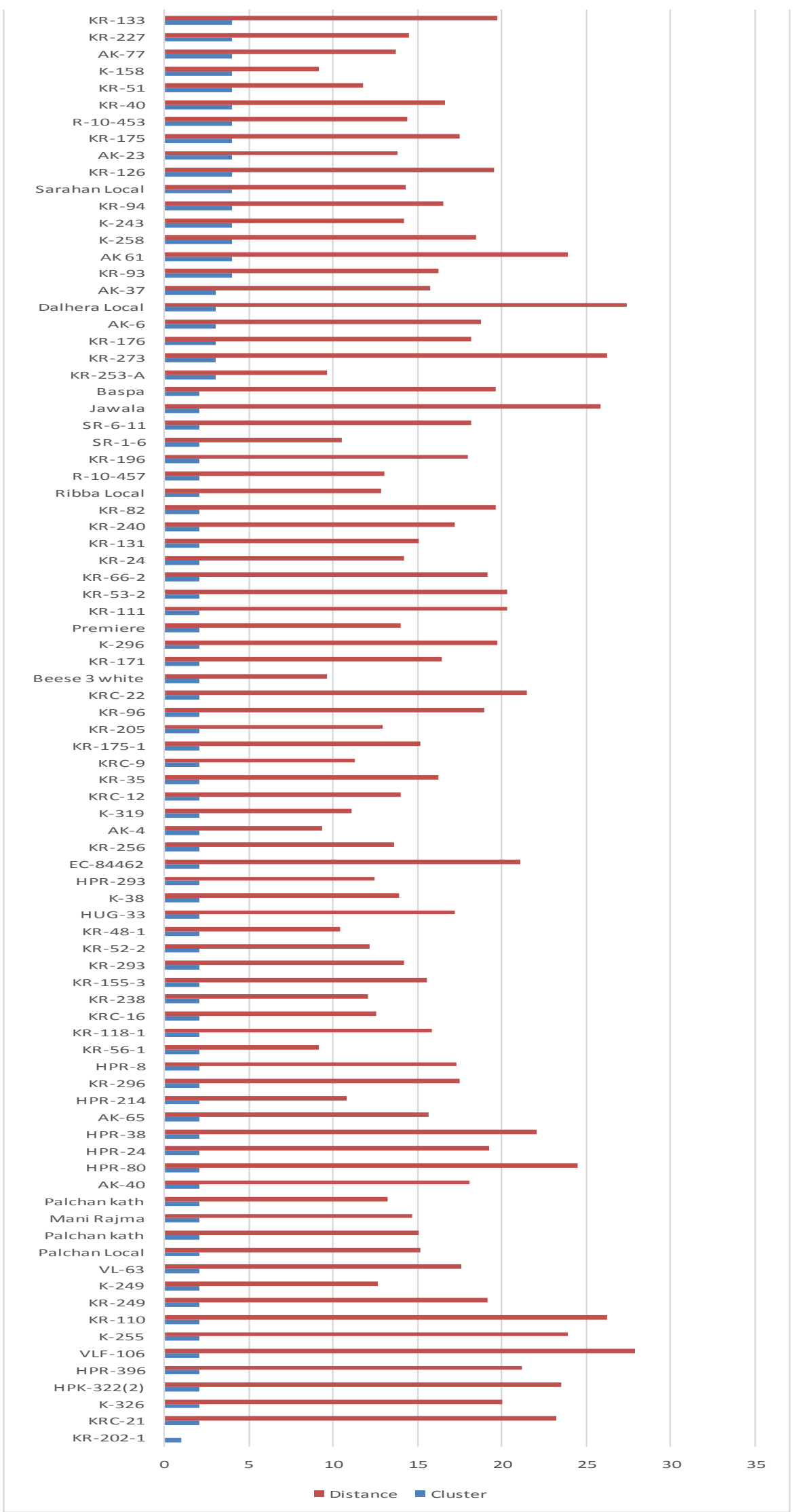




\section{Average intra and inter cluster distances}

Average intra and inter cluster distances are presented in Table 3. The genotypes which were grouped in same cluster were less divergent than the ones, which were placed in different clusters. In the present study, highest inter-cluster distance was observed between cluster I and cluster VIII (153.89), followed by cluster I and cluster IV (149.99) indicating that the genotypes from divergent clusters can be intercrossed to obtain high heterotic response and also to recover desirable transgressive segregants. Highest intra-cluster distance was only observed for cluster VI (19.65) revealed that genotypes within the same cluster were quite diverse; hence selection of parents within cluster would be effective (Fig. 1).

\section{Cluster means and contribution of individual character toward divergence}

Character mean of rajmash genotypes falling under different clusters and percent contribution to genetic divergence is presented in Table 4 and 5, respectively. Cluster I showed maximum values for number of seeds per pod and seed yield and minimum for branches per plant. Cluster II showed no maximum and minimum values for any of the trait. Cluster III showed maximum values for branches per plant, number of pods per plant and days to flowering and minimum values for none of the trait. Cluster IV showed no maximum and minimum values for any of the trait. Cluster V showed maximum value for biological yield per plant and minimum values for none of the trait. Cluster VI showed maximum values for plant height and days to maturity. Cluster VII showed no maximum and minimum values for any of the trait. Cluster VIII showed maximum values for pod length, harvest index, 100 seed weight.
The maximum contribution towards genetic divergence was exhibited by biological yield per plant (38.32\%), followed by plant height $(38.15 \%), 100$ seed weight $(12.33 \%)$, seed yield per plant $(5.80 \%)$, days to maturity (2.84\%), number of pods per plant $(1.06 \%)$, days to flowering $(0.78 \%)$, harvest index (0.67) and pod length $(0.06 \%)$. In earlier studies, Mirjana (2005) reported contribution of 100 seed weight, number of pods per plant, days to flowering, seed length towards genetic divergence in common bean. Rodino et al. (2006) observed that the number of pods per plant had the greatest effect on the genetic divergence, followed by the number of branches per plant and single plant yield whereas, in present study biological yield per plant contributed maximum towards genetic divergence followed by plant height and 100 seed weight.

\section{References}

Gepts, P. and Bliss F.A. 1988. Dissemination pathways of common bean (Phaseolus vulgaris, Fabaceae) deduced from phaseolin electrophoretic variability. Economic Botany. 42(1): 86-104.

Gepts, P., Kmiecik, K., Pereira, P. and Bliss, F.A. 1988. Dissemination pathways of common bean (Phaseolus vulgaris, Fabaceae) deduced from phaseolin electrophoretic variability. The American Economic Botany. 42(2): 7385.

Mahalanobis, P.C. 1928. On the generalized distance in statistics. In: Proceedings of the National Academy of Science (India). 2: 49-55.

Mirjana, V. 2005. Principal component analysis of dry bean collection. Bean Improvement Corporation. 48:16-17.

Rao, C.R. 1953. Advanced statistical methods in biometric research New York: John Wiley. 390 Pp. 
Rodino, A.P., Santalla, M., Gonzalez, M.A., Ron, A.M. and Singh, S.P. 2006. Novel genetic variations in common bean from the Iberian Peninsula. Crop Science. 46: 2540-2546.

Sharma, M.K., Mishra, S. and Rana, N.S. 2009. Genetic divergence in French bean (Phaseolus vulgaris L.) pole type cultivars. Legume Research. 32(3): 220-223.

Zeven, A.C. 1997. The introduction of the common bean (Phaseolus vulgaris L.) into Western Europe and the phenotypic variation of dry beans collected in The Netherlands in 1946. Euphytica. 94(4): 319-328.

Zeven, A.C., Waninge, J., Van, H.T. and Singh, S.P. 1999. Phenotypic variation in a core collection in common bean (Phaseolus vulgaris L.) in The Netherlands. Euphytica. 109(1): 93106.

\section{How to cite this article:}

Sharma, S., H.K. Chaudhary, A. Pathania and Thakur, S. 2019. Assessment of Genetic Diversity in Indian Common Bean Germplasm for Yield Traits. Int.J.Curr.Microbiol.App.Sci. 8(01): 250-260. doi: https://doi.org/10.20546/ijcmas.2019.801.028 